\title{
Combining Bias and Variance Reduction Techniques for Regression Trees
}

\author{
Yuk Lai Suen ${ }^{1}$, Prem Melville ${ }^{2}$, and Raymond J. Mooney ${ }^{3}$ \\ 1 Dept. of Electrical and Computer Engr., Univ. of Texas at Austin \\ suen@ece. utexas.edu \\ 2 Dept. of Computer Sciences, Univ. of Texas at Austin \\ melville@cs.utexas.edu \\ 3 mooney@cs.utexas.edu
}

\begin{abstract}
Gradient Boosting and bagging applied to regressors can reduce the error due to bias and variance respectively. Alternatively, Stochastic Gradient Boosting (SGB) and Iterated Bagging (IB) attempt to simultaneously reduce the contribution of both bias and variance to error. We provide an extensive empirical analysis of these methods, along with two alternate bias-variance reduction approaches - bagging Gradient Boosting (BagGB) and bagging Stochastic Gradient Boosting (BagSGB). Experimental results demonstrate that SGB does not perform as well as IB or the alternate approaches. Furthermore, results show that, while BagGB and BagSGB perform competitively for low-bias learners, in general, Iterated Bagging is the most effective of these methods.
\end{abstract}

\section{Introduction}

The decomposition of a learner's error into bias and variance terms provides a way of analyzing the behavior of different learning algorithms [1]. Various methods have been devised to reduce either the bias or variance of a learner. Some methods, such as Gradient Boosting [2], can reduce bias by increasing the expressive power of the base learner. While other methods, such as bagging [3], mainly reduce variance by subsampling the training data. There have been some attempts of combining techniques for bias and variance reduction, both for classification [4, 5] and for regression [6, 7]. For regression, Friedman [7] introduced Stochastic Gradient Boosting (SGB) as a method that reduces the variance of Gradient Boosting (GB) by incorporating randomization in the process. Breiman [6] presented a related method, Iterated Bagging (IB) that attempts to reduce the bias of bagging predictors. Despite their similarities, to our knowledge, there has been no direct experimental comparison of these two methods. In this paper, we present a detailed empirical analysis of SGB and IB. We show that IB significantly outperforms SGB when applied to both pruned and unpruned regression trees. We also explored two alternate methods for combining bias and variance reduction techniques for regression - bagging Gradient Boosting (BagGB) and bagging Stochastic Gradient Boosting (BagSGB). Our experiments show that these methods also significantly outperform SGB. In comparison to IB, BagGB and BagSGB are equally effective when applied to unpruned regression trees. However, for pruned regression trees, which have a higher bias, we observe that IB is the most effective at error reduction. This paper also 
presents a bias-variance analysis of the different algorithms, which provides a better understanding of the relative effectiveness of these methods.

Section 2 provides a brief background on the bias-variance decomposition of error. In section 3, we describe all the algorithms discussed in this paper, and our main experimental results are presented in section 4 In section 5, we discuss the results of our bias-variance analysis; and section 6 presents our future work and conclusions.

\section{Bias-Variance Decomposition of Error}

The following formulation of the bias-variance (BV) decomposition is based on [8]. Let us assume our data arose from a model $y=F(x)+\epsilon$, where the random error $\epsilon$ has $E(\epsilon)=0$ and $\operatorname{Var}(\epsilon)=\sigma_{\epsilon}^{2}$. Then the expected prediction error of a regression model $\hat{F}(x)$ for a point $x=x_{i}$ using squared-error loss can be expressed as:

$$
\begin{aligned}
\Psi\left(y, \hat{F}\left(x_{i}\right)\right) & =E\left[\left(y-\hat{F}\left(x_{i}\right)\right)^{2} \mid x=x_{i}\right] \\
& =\sigma_{\epsilon}^{2}+\left[E\left(\hat{F}\left(x_{i}\right)\right)-F\left(x_{i}\right)\right]^{2}+E\left[\hat{F}\left(x_{i}\right)-E\left(\hat{F}\left(x_{i}\right)\right)\right]^{2} \\
& =\sigma_{\epsilon}^{2}+\operatorname{bias}^{2}\left(\hat{F}\left(x_{i}\right)\right)+\operatorname{variance}\left(\hat{F}\left(x_{i}\right)\right)
\end{aligned}
$$

The first term is the irreducible error, which is the variance of the target function around its true mean $F(x)$. This error cannot be avoided no matter how well we model $F(x)$. The second term is the contribution of squared bias to error, which is the amount by which the average of our estimates differs from the true mean. The last term is the contribution of variance to error, which is the expected squared deviation of $\hat{F}\left(x_{i}\right)$ around its mean. For brevity, we will refer to the contribution of squared bias and variance to error as bias ${ }^{2}$ and variance respectively. In general, more complex models have lower bias and higher variance; e.g., unpruned decision trees tend to have low bias and high variance, while decision stumps have a very high bias but low variance.

\section{Algorithms}

\subsection{Gradient Boosting and Stochastic Gradient Boosting}

Gradient Boosting (GB) [2] is an iterative algorithm which constructs additive models by fitting a base learner to the current residue at each iteration; where the residue is the gradient of the loss function being minimized with respect to the model values at each data point. In [9], Friedman introduced Stochastic Gradient Boosting (SGB) which improves the accuracy of GB by reducing its error due to variance. In SGB, at each iteration a subsample of data is drawn uniformly at random, without replacement, from the full training set. This random subsample is used to train the base learner to produce a model for the current stage. Friedman [7] states that the idea of using a random subset of the training set at each stages originates from bootstrap sampling in bagging, and has a similar variance-reducing effect on the combined model. The SGB method (for squared-error loss) is presented in Algorithm 1 GB can be viewed as a special case of this algorithm in which the entire training set is used at each iteration, i.e., $f=1.0$. In our experiments, the shrinkage parameter $\nu$ for GB and SGB was set to 1 . 


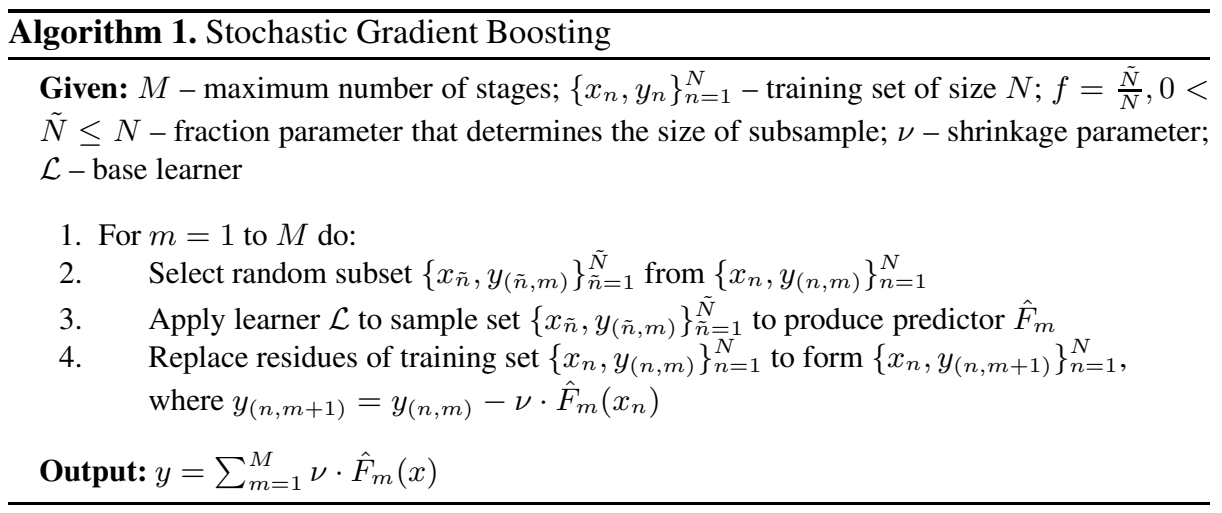

\subsection{Iterated Bagging}

Bagging has been shown to reduce the variance of predictors, while leaving the bias largely unchanged [3]. Iterated Bagging (IB) [6], also known as Adaptive Bagging [10], is an effort to reduce the bias error of the low-variance bagging predictors. Similar to $\mathrm{SGB}$, it is a stage-wise algorithm that attempts to minimize the residue in each stage. IB addresses bias and variance reduction in two ways: (1) it uses low-variance bagging predictors to compute residues and (2) it computes unbiased estimates of residues using out-of-bag calculations [10]. The outline of IB is presented in Algorithm 2] In our experiments, the threshold parameter $\tau$ for IB was set to 1.1, as done in [6].

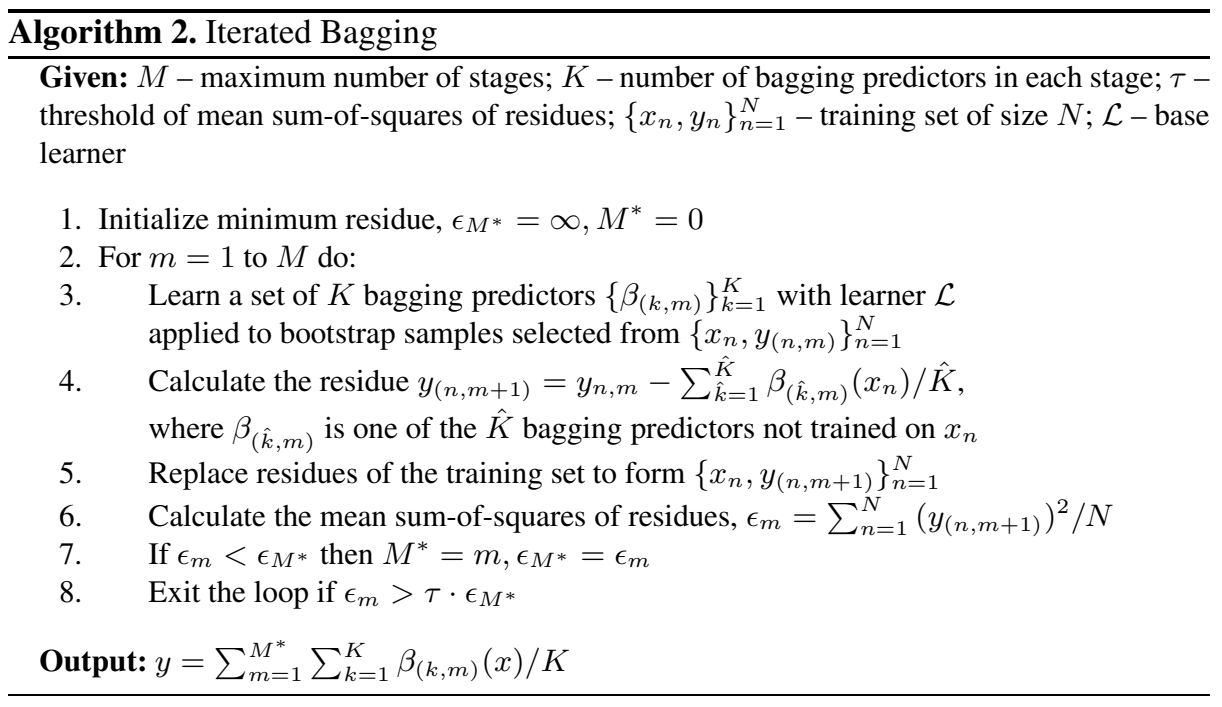

\subsection{Bagging GB and Bagging SGB}

We explored two alternative approaches to bias-variance reduction — bagging Gradient Boosting (BagGB) and bagging Stochastic Gradient Boosting (BagSGB). BagGB and 
BagSGB use GB and SGB, respectively, as the base learners in each stage of building a bagging predictor. A total of $K$ bootstrap sets of training instances are randomly selected to train $K$ GB (or SGB) predictors. The output $y$ of a test input $x$ is predicted by averaging the predictions of the $K$ base predictors.

BagGB should reduce the variance error of predictions by stabilizing the predictions of the GB base learners. BagGB and IB are similar as they both possess two components: (1) a bagging predictor to stabilize the predictions of the base learners by averaging the results of the predictors each trained with a different bootstrap sample and (2) a greedy stage-wise training of base predictors to minimize the residues. The difference between IB and BagGB is that IB performs greedy stage-wise training with a set of bagging predictors to stabilize the predictions of their base learners, while BagGB stabilizes the predictions of a set of base-predictors, each of which performs greedy stage-wise training. Although SGB already attempts to reduce the variance of GB through randomization, we believe that bagging SGB may further enhance its variance reduction.

\section{Experimental Evaluation}

\subsection{Methodology}

We ran all our experiments on 25 datasets, with continuous class (target) values, from the UCI repository [11]. Details on the datasets can found in the extended version of this paper [12]. We compared 7 different regression methods, which are listed in Table 1 along with their setup parameters. The performance of most meta-learners (additive models) varies with the number of base models used. In order to make the comparison fair, we chose parameters such that each method produces 100 base models. In the case of IB, this is an upper bound since it can choose to use fewer models. As a base learner for all the meta-learners we used M5' [13], which is regression tree induction modified based on [14] and [15]. We ran separate sets of experiments on pruned M5' and unpruned $\mathrm{M5}^{\prime}$. In pruned $\mathrm{M}^{\prime}$, the regression tree is pruned back from the leaves, so long as the expected estimated error decreases. All our results were averaged over 10 runs of 10-fold stratified cross-validation. The difference in performance between two systems was compared using a two-tailed paired t-test $(p<0.05)$.

The performance of SGB and BagSGB is dependent on the fraction parameter $f$ chosen for the experiment. Some values for $f$ perform significantly better than others on the same dataset. In order to compare with the best instances of SGB and BagSGB,

Table 1. Experimental setup of each method

\begin{tabular}{|l|l|}
\hline Algorithm & Description \\
\hline IB & 10 stages of IB with 10 stages of bagging each $(M=10, K=10)$. \\
BagSGB/BagGB & 10 stages of bagging $\times 10$ SGB/GB iterations each $(M=10)$. \\
SGB/GB & 100 iterations $(M=100)$. \\
Bagging & 100 stages of bagging M5 $5^{\prime}$ trees. \\
M5 $^{\prime}$ & pruned or unpruned M5' tree induction \\
\hline
\end{tabular}


Table 2. Summary of results comparing the different methods

\begin{tabular}{|l|lllllll|r|}
\hline & IB & BagSGB & BagGB & SGB & GB & Bag & M5 $^{\prime}$ & $\%$ ErrRed \\
\hline IB & - & $13 / 1 / 11$ & $10 / 6 / 9$ & $10 / 12 / 3$ & $18 / 4 / 3$ & $16 / 1 / 8$ & $17 / 1 / 7$ & 16.44 \\
BagSGB & $11 / 1 / 13$ & - & $10 / 8 / 7$ & $16 / 5 / 4$ & $15 / 5 / 5$ & $18 / 4 / 3$ & $21 / 2 / 2$ & 16.35 \\
BagGB & $9 / 6 / 10$ & $7 / 8 / 10$ & - & $10 / 11 / 4$ & $15 / 5 / 5$ & $16 / 2 / 7$ & $18 / 2 / 5$ & 15.61 \\
SGB & $3 / 12 / 10$ & $4 / 5 / 16$ & $4 / 11 / 10$ & - & $12 / 10 / 3$ & $13 / 1 / 11$ & $14 / 5 / 6$ & 14.39 \\
GB & $3 / 4 / 18$ & $5 / 5 / 15$ & $5 / 5 / 15$ & $3 / 10 / 12$ & - & $13 / 0 / 12$ & $13 / 1 / 11$ & 7.45 \\
Bag & $8 / 1 / 16$ & $3 / 4 / 18$ & $7 / 2 / 16$ & $11 / 1 / 13$ & $12 / 0 / 13$ & - & $17 / 5 / 3$ & 1.98 \\
M5 & $7 / 1 / 17$ & $2 / 2 / 21$ & $5 / 2 / 18$ & $6 / 5 / 14$ & $11 / 1 / 13$ & $3 / 5 / 17$ & - & - \\
\hline
\end{tabular}

(a)Base learner: unpruned $\mathrm{M5}^{\prime}$

\begin{tabular}{|l|lllllll|r|}
\hline & IB & BagSGB & BagGB & SGB & GB & Bag & M5 $^{\prime}$ & $\%$ ErrRed \\
\hline IB & - & $18 / 4 / 3$ & $20 / 3 / 2$ & $23 / 1 / 1$ & $24 / 0 / 1$ & $19 / 3 / 3$ & $22 / 1 / 2$ & 16.89 \\
BagSGB & $3 / 4 / 18$ & - & $9 / 9 / 7$ & $19 / 6 / 0$ & $18 / 6 / 1$ & $19 / 4 / 2$ & $22 / 3 / 0$ & 11.82 \\
BagGB & $2 / 3 / 20$ & $7 / 9 / 9$ & - & $18 / 7 / 0$ & $17 / 7 / 1$ & $21 / 2 / 2$ & $22 / 2 / 1$ & 11.85 \\
SGB & $1 / 1 / 23$ & $0 / 6 / 19$ & $0 / 7 / 18$ & - & $2 / 18 / 5$ & $13 / 5 / 7$ & $16 / 8 / 1$ & 8.14 \\
GB & $1 / 0 / 24$ & $1 / 6 / 18$ & $1 / 7 / 17$ & $5 / 18 / 2$ & - & $13 / 4 / 8$ & $16 / 7 / 2$ & 8.55 \\
Bag & $3 / 3 / 19$ & $2 / 4 / 19$ & $2 / 2 / 21$ & $7 / 5 / 13$ & $8 / 4 / 13$ & - & $16 / 7 / 2$ & 2.59 \\
M5 & $2 / 1 / 22$ & $0 / 3 / 22$ & $1 / 2 / 22$ & $1 / 8 / 16$ & $2 / 7 / 16$ & $2 / 7 / 16$ & - & - \\
\hline
\end{tabular}

(b)Base learner: pruned $\mathbf{M 5}^{\prime}$

we performed 10 runs of 10-fold cross-validation on SGB and BagSGB with different values of $f$ from $\{0.4,0.5,0.6,0.7,0.8,0.9\}$ and selected the $f$ that produced the lowest error for each dataset.

\subsection{Results}

Tables 2(a) and 2(b) summarize the results of our experiments using unpruned and pruned M5' base learners respectively. Each cell in the tables reports a win/draw/loss comparison between the algorithm in the row versus the algorithm in the column. The win/draw/loss record presents three values, the number of data sets for which algorithm $A$ obtained better, equal, or worse performance than algorithm $B$ with respect to rootmean-squared (RMS) error. A win or loss is only counted if the difference in values is determined to be significant at the 0.05 level by a paired $t$-test. The last column of each table presents the percentage reduction of the RMS error using different algorithms compared with using M5'. This value is averaged over all the 25 datasets, and provides an indication of the magnitude of improvements one can expect on average. In the following subsections we summarize the key comparisons from Table 2 .

IB vs. SGB: Our results show that IB significantly outperforms SGB, both in terms of win/draw/loss records and error reduction. The differences in performance are more dramatic on pruned M5', where IB performs better than SGB on 23 of the 25 datasets, and produces twice the error reduction on average. The marked performance difference on pruned $\mathrm{M5}^{\prime}$ can be attributed to IB's superior bias-reduction.

SGB, BagGB and BagSGB: BagGB performs significantly better than SGB, both for pruned and unpruned M5'. Similarly to IB, the differences are more pronounced 
on pruned M5', where BagGB wins over SGB on 18 of the datasets, with no significant losses. The results suggest that applying bootstrap sampling to GB has a better variance-reducing effect than the randomization incorporated in SGB. In fact, applying bagging to SGB (BagSGB), can significantly drive down the error of SGB, as can be seen for both M5' settings. BagSGB performs marginally better than BagGB in terms of win/draw/loss records, though their error reductions are quite comparable.

IB vs. BagGB/BagSGB: On unpruned M5', BagGB and BagSGB perform comparably to IB both in terms of win/draw/loss records and error reduction - all methods producing approximately a $16 \%$ reduction in RMS error. However, for pruned $\mathrm{M}^{\prime}$ trees, which have higher bias, IB exhibits a significant advantage over BagGB and BagSGB. It wins over BagGB and BagSGB on 20 and 18 datasets respectively. We also observe approximately a $5 \%$ difference in error reduction between IB and the other methods. IB's effectiveness at debiasing learners makes it a clear winner in higher bias settings.

SGB vs GB: Our results on high-variance unpruned M5' support the claim in [7] that SGB has a better variance-reducing effect than GB. SGB on average reduced $14.39 \%$ of the error of unpruned $\mathrm{M5}^{\prime}$, while GB reduced only $7.45 \%$. However, SGB has significant wins in only 12 datasets and ties with GB in 10. Although, the error reduction of SGB is quite good, the win/draw/loss results do not suggest as significant an advantage of SGB over GB as in [9]. In fact, on pruned M5', the performance of SGB and GB are tied on 18 datasets, with SGB performing slightly worse on the other datasets.

Bias-variance reduction vs. bias or variance reduction: $G B$ and bagging focus solely on reducing the bias or the variance of learners. On the other hand, IB, SGB, BagGB and BagSGB attempt to reduce both the contribution of bias and variance to error. For brevity, we will refer to these four methods as BV-methods. Our results show that generally the BV-methods have a significant advantage over GB and bagging, even when using the same number of base models. When compared to GB, BV-methods perform significantly better on at least 12 datasets and lose on at most 5 datasets. The only exception is SGB using pruned M5', which loses to GB by a margin of 3 datasets. Even when compared to bagging, SGB is less effective than the other BV-methods. It wins by a margin of 2 (13 wins vs. 11 losses) and 6 (13 wins vs. 7 losses) when using unpruned M5' and pruned M5' respectively. The other BV-methods win by at least 16 datasets and lose on at most 8 when compared to bagging. The results clearly indicate that combining techniques for bias and variance reduction is more effective than focusing on either component alone.

\section{Bias-Variance Analysis}

We explain most of our results based on how the different learners effect the bias and variance components of the error. To support our conjectures, we ran additional experiments to explicitly measure the bias and variance reducing effects of the methods presented. As in [6], we performed BV decompositions on three synthetic datasets Friedman 1, 2 and 3 [1. We do not introduce noise in these datasets, so that the evalua-

\footnotetext{
${ }^{1}$ Details of the datasets can be found in [12].
} 
Table 3. Methods in order of increasing bias, variance and overall error

\begin{tabular}{|l|l|l||l|l|l||l|l|l|}
\hline \multicolumn{3}{|c||}{ Friedman1 } & \multicolumn{3}{|c||}{ Friedman2 } & \multicolumn{3}{|c|}{ Friedman3 } \\
\hline Bias $^{2}$ & Var. & Err. & Bias $^{2}$ & Var. & Err. & Bias $^{2}$ & Var. & Err. \\
\hline IB & Bag & IB & IB & IB & IB & IB & Bag & IB \\
GB & BagSGB & BagSGB & GB & Bag & BagSGB & GB & BagSGB & BagGB \\
SGB & BagGB & BagGB & BagGB & BagSGB & BagGB & SGB & IB & GB \\
BagGB & IB & GB & SGB & BagGB & GB & BagGB & BagGB & BagSGB \\
BagSGB & M5 & SGB & BagSGB & GB & SGB & BagSGB & M5' & SGB \\
M5 & GB & Bag & M5 & M5 & Bag & M5 & SGB & Bag \\
Bag & SGB & M5 & Bag & SGB & M5 & Bag & GB & M5' \\
\hline
\end{tabular}

tion of the bias and variance reduction capability of a learner is not confounded with its ability to handle noise. We use synthetic datasets, so that we can control for noise and get better estimates of bias and variance.

To estimate bias and variance we used the method proposed by Kohavi and Wolpert [16], appropriately modified for regression (as opposed to classification). Each dataset was divided into two halves, $D$ and $E$. $D$ was used to draw our sample of training sets from, and $E$ was used to estimate the terms in the $\mathrm{BV}$ decomposition. We generated 50 training sets from $D$ sampled uniformly at random without replacement. Each training set of size 200 was selected from the pool of 400 examples in $D$. Each learning algorithm was run on each of the training sets and the squared bias and variance terms were calculated on set $E$ based on equation 1 . These values were averaged over all 50 train-test cycles. For SGB and BagSGB, we used a fraction $f=0.6$; which is roughly equivalent to drawing bootstrap samples at each iteration.

Table 3 presents the different algorithms applied to unpruned $\mathrm{M}^{\prime}$, in the order of increasing bias, variance and overall error on each dataset. The results for pruned trees were qualitatively similar, though in general the errors were higher for all methods. For detailed results see [12]. We observe that GB performs very well at reducing bias, but does not perform well at variance reduction. In fact, on Friedman1 and Friedman3, GB actually increases the variance of the base learner. Analogously, bagging can increase the bias of the learner, but performs very well in terms of variance reduction. By combining the power of bagging and GB, BagGB produces a lower overall error than each of its components. Similarly, BagSGB improves on the bias reduction of bagging and the variance reduction of SGB, and as a result produces a lower overall error than both component algorithms. IB shows the best performance on overall error, and it appears to be quite effective in reducing both bias and variance. In fact, IB also performs the best in terms of bias reduction on all three datasets.

\section{Future Work and Conclusion}

We compared four approaches to combining bias and variance reduction techniques Stochastic Gradient Boosting, Iterated Bagging, bagging Gradient Boosting and bagging Stochastic Gradient Boosting. Our results demonstrate that methods for combining bias and variance reduction (BV-methods) are more effective than methods that focus either on bias or variance in isolation. We also showed that while SGB often improves 
on GB, it is not very consistent and is easily outperformed by the other BV-methods. Experimental results show that for unpruned trees, which are low-bias learners, BagGB and BagSGB perform somewhat comparably to IB. However, IB, being a more effective bias-reduction method, performs much better compared to other algorithms when applied to pruned trees.

In our study, we restricted our methods to building at most 100 models each. Typically, the performance of these ensemble methods (or additive models) improve with ensemble size. In future work, we would like to explore the relationship between the number of models used and the effectiveness of each method. All our experiments were run on UCI datasets, commonly used in previous studies. However, these datasets are not very large - the largest has 625 instances. It would be good to see how results vary for much larger datasets. Experimenting with base learners other than decision trees, such as neural networks and support vector machines would also be very useful.

\section{Acknowledgments}

We like to thank Horris Tse for assistance with running some of the experiments. Prem Melville and Raymond Mooney were supported by DARPA grant HR0011-04-1-007.

\section{References}

[1] Geman, S., Bienenstock, E., Dorsat, R.: Neural networks and the bias/variance dilemma. Neural Computation 4 (1992) 1-58

[2] Friedman, J.: Greedy function approximation: a gradient boosting machine. Technical report, Stanford University Statistics Department (1999)

[3] Breiman, L.: Bagging predictors. Machine Learning 24 (1996) 123-140

[4] Valentini, G., Dietterich, T.G.: Low bias bagged support vector machines. In: Proc. of 20th Intl. Conf. on Machine Learning (ICML-2003), Washington, DC (2003) 752-759

[5] Webb, G.: Multiboosting: A technique for combining boosting and wagging. Machine Learning 40 (2000) 159-196

[6] Breiman, L.: Using iterated bagging to debias regressions. Machine Learning 45 (2001) 261-277

[7] Friedman, J.: Stochastic gradient boosting. Technical report, Stanford University Statistics Department (1999)

[8] Hastie, T., Tibshirani, R., Friedman, J.: The Elements of Statistical Learning. Springer Verlag, New York (2001)

[9] Friedman, J., Hastie, T., Tibshirani, R.: Additive logistic regression: a statistical view of boosting. Technical report, Stanford University Statistics Department (2000)

[10] Breiman, L.: Using adaptive bagging to debias regressions. Technical report, UC Berkeley Statistics Department (1999)

[11] Blake, C.L., Merz, C.J.: UCI repository of machine learning databases. http://www.ics.uci.edu/ mlearn/MLRepository.html (1998)

[12] Suen, Y.L., Melville, P., Mooney, R.J.: Combining bias and variance reduction techniques for regression. Technical Report UT-AI-TR-05-321, University of Texas at Austin (2005) www.cs.utexas.edu/ ml/publication. 
[13] Wang, Y., Witten, I.: Inducing model trees for continuous classes. ECML Poster Papers (1997) 128-137

[14] Quinlan, J.: Learning with continuous classes. In: Proceedings of 5th Australian Joint Conference on Artificial Intelligience. (1992) 343-348

[15] Breiman, L., Friedman, J.H., Olshen, R., Stone, C.: Classification and Regression Trees. Wadsworth and Brooks, Monterey, CA (1984)

[16] Kohavi, R., Wolpert, D.H.: Bias plus variance decomposition for zero-one loss functions. In Saitta, L., ed.: Proc. of 13th Intl. Conf. on Machine Learning (ICML-96), Morgan Kaufmann (1996) 